\title{
Innovative Blood Tests for Hepatocellular Carcinoma: Liquid Biopsy and Evaluation of Systemic Inflammatory Reaction
}

\author{
Ilze Strumfa, Dzeina Mezale, Boriss Strumfs, \\ Andrejs Vanags, Arturs Kalva, Dainis Balodis, \\ Ilze Fridrihsone, Arnis Abolins and Janis Gardovskis
}

Additional information is available at the end of the chapter

http://dx.doi.org/10.5772/intechopen.76599

\begin{abstract}
Hepatocellular carcinoma (HCC) is an aggressive tumour associated with dismal prognosis. To improve the outcome, early diagnostics is important. At present, classical HCC diagnostics is based on evaluation of risk factors, surveillance in cirrhotic patients, preference for non-invasive diagnosis by computed tomography or magnetic resonance imaging and biopsy confirmation in controversial cases. However, ambiguous radiological presentation, biopsy-related complications or insufficient representation of the pathology in the tissue core are well-known problems. Panel assessment of microRNAs has diagnostic and prognostic value; thus, in future, microRNA-based liquid biopsy could partially reduce the need for core biopsies. Systemic inflammatory reaction (SIR), characterised mainly by neutrophil-to-lymphocyte ratio, platelet-to-lymphocyte ratio and Glasgow prognostic score, may have prognostic value and can be incorporated in criteria for certain treatment approaches, e.g., becoming an adjunct to Milan criteria. Thus, innovations in HCC diagnostics are expected in the field of miRNA-based liquid biopsy for diagnosis/prognosis and SIR for prognosis/selection of treatment.
\end{abstract}

Keywords: hepatocellular carcinoma, HCC, liquid biopsy, miRNA, systemic inflammatory response, neutrophil-to-lymphocyte ratio, platelet-to-lymphocyte ratio, Glasgow prognostic score, prognosis 


\section{Introduction}

Hepatocellular carcinoma is one of the most aggressive human cancers. The total oncological mortality is decreasing in many developed countries, e.g., it has been reduced by $23 \%$ since 1991 in the United States of America (USA). In contrast, death rate of HCC is increasing, along with the incidence of this tumour [1,2]. Positive changes are expected due to risk factor eradication by vaccination against hepatitis $B$ and improved treatment of chronic hepatitis $C$. The treatment of HCC has also developed significantly, including radiofrequency ablation, transarterial chemoembolisation, liver resection and transplantation as well as molecular targeted treatment by sorafenib. However, SEER-based analysis has revealed that survival has improved in early but not in advanced cases [2]. Thus, timely diagnostics remain an important goal.

Most of the hepatocellular carcinoma cases develop on the background of liver cirrhosis or chronic inflammatory liver disease in precirrhotic stage, e.g. chronic viral hepatitis B or C, alcohol-induced or autoimmune liver disease or non-alcoholic steatohepatitis (NASH). This might facilitate the diagnostics by screening of the risk population. Nowadays, screening by ultrasonography and non-invasive radiological diagnosis by the means of computed tomography or magnetic resonance imaging is the mainstay of HCC diagnostics. However, the radiological findings in early cases can be difficult to interpret. Biopsy is indicated in such controversial cases.

However, the biological course of HCC can result in diagnostic difficulties even in biopsy. HCC frequently develops in a dysplastic cirrhotic nodule. Such early HCC is typically well differentiated. Over time, it progresses to advanced dedifferentiated HCC. The resulting heterogeneity can lead to diagnostic problems and failures in biopsy due to sampling errors. For instance, if a small nodule seemed suspicious but not overtly malignant by radiological imaging, leading to biopsy, the differential diagnosis between dysplastic nodule and HCC will frequently imply the necessity to distinguish between premalignant process and welldifferentiated tumour, usually lacking marked cell atypia or clear-cut invasion. In addition, both processes can be adjacent in the tissues. Consequently, early diagnostics of HCC is not straightforward even in biopsy.

In addition, biopsy can cause complications, including arterial hypotension, bleeding, pneumothorax, haemothorax, haemobilia, acute pancreatitis, visceral perforation, biliary fistulas, sepsis and needle breakage. Arterial hypotension is seen frequently (1.1-4.0\%), mostly due to vasovagal reaction. In few cases, falling arterial blood pressure might indicate bleeding, if it is unusually severe. Bleeding can develop in the liver tissues or in the peritoneal cavity. It is seen in $4.5 \%$ of patients and is more frequent if INR is elevated: frequency of bleeding was $3.3 \%$ in patients having INR 1.3-1.5 and 7.1\% among those who had INR > 1.5. Pneumothorax and haemothorax have been reported in 0.35 and $0.18 \%$ of patients, if the needle has accidentally passed through lung or diaphragmatic and intercostal blood vessels. Haemobilia has been described in $0.1 \%$ of patients and can induce acute pancreatitis via biliary obstruction. The frequency of visceral perforation, biliary fistulas, sepsis and needle breakage is $0.01 \%$ each. In addition, biopsy can result in pain, experienced in up to $84 \%$ of patients. In $40 \%$ of cases, pain lasts for 24 hours. It is attributable to skin and liver capsule damage, viscerosomatic irradiation 
(to shoulder) or complications that lead to peritoneal irritation (bleeding, bile accumulation in the peritoneum perforation of the internal organs). Few death cases have been reported after liver biopsy: $0.01-0.1 \%$ of patients [3].

Non-invasive means of HCC diagnostics would be beneficial. Two promising pathways include so-called liquid biopsy by microRNA analysis in blood and assessment of tumourinduced systemic inflammatory reaction (SIR). MicroRNA spectrum might have diagnostic and prognostic value. Regarding SIR, prognostic aspects have been studied and appear as promising adjunct how to select patients for treatment.

\section{2. miRNAs in the diagnostics of hepatocellular carcinoma}

MicroRNAs (miRNAs) are small, double-stranded, non-coding RNA molecules consisting of approximately 22 nucleotides. MiRNAs regulate gene expression at the post-transcriptional level $[4,5]$ acting as large-scale molecular switches. MiRNAs are found not only in cells but also in body fluids. Due to the stable and relatively simple structure, these molecules are good biomarkers for diagnostic and prognostic evaluation complying with the idea of so-called liquid biopsy - a patient-friendly blood test bringing the same information as a biopsy. In order to increase the diagnostic value of such tests, panels of miRNAs have been advocated. However, the biological course of HCC cause a possible pitfall in the elaboration of such diagnostic tests: as HCC mainly arise on the background of liver cirrhosis, inflammatory or metabolic liver diseases, these preceding pathologies can also alter the levels of miRNAs.

MiRNA-122 is attractive for its wide expression in liver tissues suggesting significant role in liver functions. MiRNA-122 is upregulated in serum of HCC patients and downregulated in HCC tissues suggesting specific excretion of miRNA-122 in blood by HCC. Although miRNA122 shows high specificity and sensitivity for HCC in comparison with healthy controls (83.3 and $81.6 \%$, respectively), levels of miRNA-122 change in other liver pathologies as well, limiting the diagnostic application [6].

Comparing HCC patients with those having hepatitis B or liver cirrhosis, serum levels of exosomal miRNA-18a, miRNA-221, miRNA-222 and miRNA-224 were increased, while miRNA101, miRNA-106b, miRNA-122 and miRNA-195 were decreased. MiRNA-16 was decreased in HCC, and the levels significantly differed from those found in hepatitis $\mathrm{C}$ virus (HCV) infection because chronic viral hepatitis $C$ and non-alcoholic fatty liver disease are characterised by the contrary changes - increase in miRNA-16. MiRNA-21 is characterised by positive characteristics in meta-analysis showing specificity and sensitivity for HCC diagnosis of 84.8 and $81.2 \%$, respectively. The changes of miRNA-21 serum levels in HCC patients significantly differ from cases of chronic hepatitis; however, other malignant tumours can also yield higher serum concentration of miRNA-21 [7].

Several panels of miRNAs have been recommended. Exploring nine serum miRNAs (miRNA21, miRNA-30c, miRNA-93, miRNA-122, miRNA-125b, miRNA-126, miRNA-130a, miRNA193b and miRNA-222) in HCC and chronic viral hepatitis C patients, nine markers were decreased in chronic hepatitis $\mathrm{C}$ versus healthy controls, while seven markers (miRNA-21, 
miRNA-30c, miRNA-93, miRNA-122, miRNA-125b, miRNA-130a and miRNA-222) were significantly $(\mathrm{p}<0.05)$ decreased in HCC versus chronic viral hepatitis $\mathrm{C}$ patients and four (miRNA-93, miRNA-122, miRNA-125b and miRNA-130a) in HCC versus non-HCC patients [8]. Panel of three miRNAs (upregulated miRNA-92a-3p, downregulated miRNA-3126-5p and upregulated miRNA-107) could discriminate HCC from healthy controls [9] Evaluating serum levels of 13 miRNAs in HCV-associated chronic hepatitis, liver cirrhosis and HCC versus healthy controls, panel of three miRNAs (miRNA-122, miRNA-885-5p and miRNA-29b) in association with serum alpha-fetoprotein (AFP) level could identify HCC versus healthy persons, while four miRNAs (miRNA-122, miRNA-885-5p, miRNA-221 and miRNA-22) and AFP were recommended for HCC diagnostics in liver cirrhosis and two (miRNA-22, miRNA-199a-3p) along with AFP - in chronic hepatitis [10]. In another study of HCV-infected patients including cases of HCV-related chronic hepatitis, liver cirrhosis and HCC, serum levels of miRNA-126, miRNA-129, miRNA-155, miRNA-203 and miRNA-223 were significantly decreased in HCC versus non-HCC patients [11]. Panel of eight miRNAs was assessed in hepatitis B virus-infected patients diagnosed with HCC or liver cirrhosis as well as in healthy controls. The levels of hsa-miRNA-206, hsa-miRNA-141-3p, hsa-miRNA-433-3p and hsamiRNA-1228-5p were significantly increased in HCC versus control group comprising both healthy and cirrhosis patients, while hsa-miRNA-199a-5p, hsa-miRNA-122-5p, hsa-miRNA192-5p and hsa-miRNA-26a-5p were downregulated [4].

In addition to the diagnostic role, miRNAs have been evaluated in the prognostic aspect. The influence of miRNAs upon HCC stem cells has been exploited. It has also been suggested that miRNAs could become treatment targets [12].

\section{Systemic inflammatory response}

Many tumours, including hepatocellular carcinoma, evoke systemic inflammatory reaction (SIR). In the recent years, cancer-induced SIR has become an attractive research area as changes in blood cell counts or ratios or blood levels of certain proteins are associated with the biological potential, course and treatment response in many malignant tumours. Although complex pathogenesis lies behind these changes, SIR can be evaluated by simple, widely available and economically feasible blood tests.

SIR in cancer patients develops through local and central mechanisms. Locally, the invasive growth of malignant tumour injures surrounding parenchyma, connective tissues and endothelium. The tissue damage leads to inflammation necessitating supply of inflammatory cells from the bone marrow through the circulation. The production of acute phase proteins becomes upregulated as well. Endothelial injury activates platelets; indeed, the association between hypercoagulation and advanced cancers is classical. Tumour necrosis and hypoxia are additional causes of local inflammatory response. Cancer can also evoke immune response manifesting by local cellular reactions. In turn, the inflammation can have both tumour-enhancing and tumour-suppressing outcomes. The released cytokines and transcription factors can upregulate the proliferation of malignant cells. Release of metalloproteinases and other enzymes can promote tissue degradation facilitating invasion. Angiogenesis can be upregulated as well. The immune system, in turn, can limit growth of the tumour. 
The systemic effects of cancer include alterations in bone marrow function, especially myelopoiesis. Besides the increased production and release of leukocytes, immature myeloid cells, including the precursors of granulocytes and monocytes, are retained in early stages of differentiation. Immature myeloid cells can act as immune suppressors and generate pre-metastatic niches, among other pathogenetic processes [13]. Thus, it has even been stated that cancer is an inflammatory disease [14]. Further, neutrophils can form neutrophil extracellular traps developed from externalised DNA network. These nets are bidirectionally associated with platelet activation and can contribute to cancer progression via several mechanisms; therefore, neutrophil extracellular traps also represent an attractive treatment target [15].

Cancer-related SIR involves cells of innate and adaptive immunity as well as soluble factors. Macrophages are recruited in tumour by hypoxia and tumour-released molecular agents including growth factors and cytokines [16]. Macrophage phenotype switch from tumoursuppressing classical M1 to tumour-promoting M2 subtype promotes angiogenesis and immunosuppression. Platelet activation contributes to cancer progression and patient mortality [15]. Neutrophils are locally recruited in the cancer via chemokine signalling. Neutrophil activation can contribute to angiogenesis and increased blood vessel permeability locally and metastatic spread systemically. In addition, immature myeloid cells and neutrophil extracellular traps might have tumour-promoting activity. These molecular events also highlight the association between infection or surgery-induced inflammation [17] and cancer relapse or metastatic spread. Thus, innate immunity is generally thought to act as tumour enhancers. In contrast, lymphocytes representing the adaptive immunity are considered to have tumour-suppressing effects [16], although contrary effects have been ascribed to certain subpopulations [18].

HCC can be considered a classical inflammation-induced cancer, as its most common risk factors are hepatitis B and hepatitis $C$ virus infections. Inflammation is also present in liver tissues in patients affected by alcohol-induced hepatitis or NASH. Thus, SIR is not expected to have diagnostic value. Indeed, SIR parameters change before the tumour develops, e.g., increased NLR has been observed in chronic viral hepatitis C [19]. NLR is an independent prognostic factor in liver cirrhosis [20].

Wide variety of inflammation-based markers could be used as indicators of HCC prognosis, tumour recurrence and response to specific treatment. In particular, neutrophil, lymphocyte and platelet counts as well as C-reactive protein and albumin and their combinations, neutrophil-to-lymphocyte ratio (NLR), platelet-to-lymphocyte ratio (PLR) and Glasgow prognostic score (GPS) have prognostic value.

\subsection{Neutrophil-to-lymphocyte ratio in HCC}

Neutrophil-to-lymphocyte ratio is the most extensively evaluated parameter of SIR in HCC. NLR, calculated as the ratio between count of neutrophils and lymphocytes in blood, seems to reflect the intensity of systemic pro- and anti-tumour reaction. NLR has shown prognostic importance in surgically treated HCC cases, including early stage tumours; in liver transplantation; sorafenib treatment and different ablation techniques.

Several meta-analyses have been devoted to NLR in HCC patients. Thus, baseline NLR was associated with overall survival and recurrence-free survival, while post-treatment NLR was significantly associated with overall survival [21]. In meta-analysis of NLR in HCC patients 
treated by liver transplantation, significant association with overall and recurrence-free survival was found [22]. In an early study, NLR was already confirmed as a prognostic factor in HCC. NLR was associated with overall and disease-free survival both generally and after different treatment approaches [23].

\subsection{Neutrophil-to-lymphocyte ratio in surgically treated HCC}

In HCC patients subjected to curative resection, several research groups have identified high NLR as an independent, significant predictive factor, associated with worse overall survival [24-27], shorter recurrence-free survival [24, 25, 28] and higher frequency of recurrence [29].

The studies show some variability in design, group size and cut-off level. However, the reported differences in survival are not only statistically significant but also biologically important. In $672 \mathrm{HCC}$ patients treated by surgical resection, the recurrence rate within 1, 3 and 5 years was $77.4,55.2$ and $44.8 \%$ in those having low NLR $(\leq 2.5)$, while the corresponding recurrence rates were $64.1,45.2$ and $35.5 \%(p=0.016)$ in high NLR group [29]. In 303 patients who underwent surgical liver resection for HCC, high NLR $(>2.0$, based on ROC-detected cut-off) was significantly associated both with shorter recurrence-free and overall survival (both $\mathrm{p}<0.001$ ), but multivariate analysis confirmed it as an independent prognostic factor only for overall survival (hazard ratio (HR) 1.724; 95\% confidence interval (CI): 1.241-2.394; $\mathrm{p}=0.001$ ). Huang et al. evaluated 1659 HCC patients, stratifying them by NLR quartiles. The 5 -year overall survival rate was $60 \%$ in the lowest quartile contrasting with $27 \%$ in the highest quartile. NLR was associated by HR of mortality 1.031; 95\% CI: 1.002-1.060; p $=0.033$ [30]. In a large Japanese study, enrolling 958 patients who underwent hepatectomy, the 5-year survival rate was $72.9 \%$ in low NLR $(<2.81)$ group versus $51.5 \%$ in patients with high NLR [24]. In another 256 Japanese patients, NLR was confirmed by multivariate analysis as an independent prognostic factor both for overall and recurrence-free interval. The respective hazard ratios were 2.59; 95\% CI: 1.56-4.31; $\mathrm{p}<0.001$ and 2.11; 95\% CI $=1.44-3.11 ; \mathrm{p}<0.001$ [25]. In a smaller study of 113 patients, the recurrence-free survival was 42.4 months in patients having NLR $<3$ but 7.9 months in those having NLR $\geq 3.0$. The respective HR was $2.58 ; p=0.002$ [28].

The findings in Western patients have been less positive. In the largest Western series comprising 370 patients, treated in Memorial Sloan Kettering Cancer Center in New York, USA, PLR, but not NLR, was independently associated with worse recurrence-free survival and overall survival [31].

NLR retains prognostic value in several subgroups of surgically treated patients, including early cases. By multivariate analysis, preoperative NLR (at cut-off 2.8) was the strongest independent prognostic factor for overall survival after liver resection with curative intent for TNM stage I HCC. The HR was 2.69; 95\% CI: 1.57-4.59; p < 0.001. The 5-year survival in high versus low NLR group was 45.0 versus $76.4 \%$; $<<0.001$. Interestingly, the association with survival in stage II or III was not significant, reflected by $p=0.283$ and $p=0.155$. In stage I patients, high NLR predicted more frequent extrahepatic recurrence $(p=0.006)$. As growth of HCC is associated with grade progression, these results suggest that NLR reflects the biological potential of HCC [32]. In Chinese cohort of 222 patients, preoperative NLR, using cut-off at 
2.1, predicted overall survival in solitary small $(\leq 5 \mathrm{~cm}) \mathrm{HCC}$ after surgical resection and could discriminate outcome in patients having AFP levels not exceeding $400 \mathrm{ng} / \mathrm{mL}$ [33]. Further, in a large study of 963 HCC patients treated by potentially curative surgical resection, high NLR (>2.81) was an independent risk factor for overall and recurrence-free survival (both $\mathrm{p}<$ $0.001)$ in the general group as well as in early or intermediate stage HCC: Barcelona Clinic Liver Cancer (BCLC) stages 0/A or B (both $\mathrm{p}<0.05$ ) while no association was found in stage C [34]. NLR was also not associated with early $(<1$ year) mortality from cancer recurrence after liver resection for huge (at least $10 \mathrm{~cm}$ in diameter) HCC in 166 patients [35]. NLR was an independent factor that predicted $(p=0.029)$ early recurrence after curative resection of HCC presenting as a single focus in 193 Japanese patients [36]. However, contrary results have been reported as well, e.g., NLR had no prognostic significance in early HCC (BCLC stage 0/A) treated by surgical resection in 324 patients [37].

Somewhat contrasting data are reported regarding NLR in surgically treated patients with more advanced HCC. Although limited significance of NLR was previously noted in highstage or large HCC, some authors have found significant role of NLR in advanced cases. Thus, in 81 hepatectomy-treated HCC patients with portal or hepatic vein tumour thrombosis, high NLR (defined as >2.9) was an independent prognostic factor for worse overall survival, characterised by HR 1.866; 95\% CI: 1.048-3.322; $p=0.034$. Significant association with recurrencefree interval was found as well. The overall survival in high versus low NLR groups was 6.2 months versus 15.7 months; $\mathrm{p}=0.007$, while the recurrence-free survival was 2.2 versus 3.7 months; $\mathrm{p}=0.039$ [38].

Not only baseline NLR but also the dynamic changes of NLR were found to be important. In 189 patients treated by curative resection for small HCC, the temporal change of NLR was an independent prognostic factor for overall (HR 2.637; 95\% CI: 1.356-5.128; p = 0.004) and recurrence-free (HR 2.372; 95\% CI: 1.563-3.601; $\mathrm{p}<0.001$ ) survival. The 1-, 3- and 5-year survival was $92.7,70.0$ and $53.0 \%$ in patients experiencing NLR increase versus $96.2,87.5$ and $75.9 \%$ in those with decreasing NLR ( $p=0.003$ ). High preoperative or postoperative NLR had lower prognostic value in this study [39]. Paralleling observations have been reported by Hung et al., studying patients with HCC recurrence after liver resection. The 5 -year post recurrence survival was better $(45.9 \%)$ in those continuously having NLR $\leq 2.5$. Patients who had low NLR $(\leq 2.5)$ at resection but high level $(>2.5)$ at recurrence had 5-year survival of only $24.6 \%$; the difference was significant as reflected by $p=0.013$ [29].

\subsection{Neutrophil-to-lymphocyte ratio in patients receiving transplantation for HCC}

Different aspects of NLR have been evaluated in HCC patients subjected to liver transplantation, including transplantation from living donor. The prognostic value of NLR was revealed already in the early studies. Thus, NLR (applying the cut-off level at 5.0) was an independent predictor of overall and recurrence-free survival in 219 Italian patients after liver transplantation for HCC [40].

By univariate analysis, preoperative NLR was significantly associated by disease-free survival and overall survival in HCC patients after liver transplantation. The 1-, 3- and 5-year overall 
survival rate in high versus low NLR group was $81.3,56.7$ and 51.0 versus $90.9,74.2$ and $66.8 \%$ $(\mathrm{p}=0.041)$. Similarly, the 1-, 3- and 5-year recurrence-free survival rate in high versus low NLR group was $65.3,48.5$ and 39.4 versus $80.0,68.0$ and $65.2 \%$. The difference was also significant as reflected by $\mathrm{p}=0.013$ [41]. Further, NLR was an independent prognostic factor for overall and recurrence-free survival after liver transplantation for HCC as was shown by multivariate analysis of 160 Western patients [42]. NLR was proved to be an independent risk factor for overall survival $(p<0.001)$ and recurrence-free survival $(p=0.003)$ in 248 male patients treated by liver transplantation [43]. Harimoto et al. reported on 213 patients receiving living donor liver transplantation for HCC. High preoperative NLR $\geq 2.66$ was an independent predictor of recurrence [44].

NLR can be used in prognostic models to identify patients who exceed Milan criteria but still have good overall and tumour-free survival. In study reported by Wang et al., male patients were enrolled and thus the proposed models were verified in males only [43]. Combination of NLR and Hangzhou criteria has been suggested to identify patients who can be successfully treated by liver transplantation [45]. NLR has been included in the MORAL scores to predict recurrence after liver transplantation, and these scores were superior to Milan criteria [46]. Complex evaluation of NLR along with fibrinogen increases the prognostic accuracy in order to predict disease-free survival and overall survival in HCC patients treated by liver transplantation [41]. Pretransplantation NLR along with levels of C-reactive protein has been combined with Milan criteria to develop new selection criteria for living donor liver transplantation beyond Milan criteria [47].

However, contrasting findings have been published as well. Thus, NLR did not predict posttransplantation recurrence or worse overall survival in 150 patients within Milan criteria [48]. Limited prognostic impact of NLR was found in 124 patients who underwent living donor liver transplantation [49]. In Western patients, NLR was not predictive of treatment success regarding liver transplantation or other tested approaches (hepatectomy, transarterial chemoembolisation). Although the group was quite small (75), Child-Pugh and Model for End Stage Liver Disease (MELD) scores were informative [50].

\subsection{Neutrophil-to-lymphocyte ratio in sorafenib-treated HCC patients}

In patients with unresectable HCC, treated by multikinase inhibitor sorafenib, high NLR (>3.1) was a significant independent prognostic factor, associated with worse overall survival. Better treatment response was observed in patients with low NLR [51]. The findings were confirmed by another study, reporting on 442 sorafenib-treated patients (Japan, Italy and United Kingdom) with advanced HCC. High NLR again was an independent prognostic factor, predicting shorter survival with HR 1.218; 95\% CI: 1.108-1.322; $\mathrm{p}<0.0001$ [52].

Regarding combined approach, high pre-treatment NLR $(>3.0)$ was an independent predictor of worse overall survival in 40 patients with unresectable HCC treated by transcatheter arterial embolisation and sorafenib. The median survival in high versus low NLR group was 14 months $(95 \% \mathrm{CI}=10.1-17.9)$ versus 26 months $(95 \% \mathrm{CI}=17.4-34.6)$. The difference was significant $(\mathrm{p}=0.001)$ and biologically remarkable [53]. Other researchers have also confirmed that NLR was independent predictor of overall survival in patients with advanced HCC treated by sorafenib [54]. 


\subsection{Neutrophil-to-lymphocyte ratio in HCC patients undergoing tumour ablation}

NLR has been investigated in regard to different embolisation and ablation techniques. High NLR $(>3)$ predicted significantly worse treatment results $(\mathrm{p}=0.014)$ and early disease progression $(\mathrm{p}<0.0001)$ in 86 treatment-naive patients subjected to arterial chemoembolisation or radioembolisation [55]. Elevated pre-treatment NLR $(>1.85)$ was associated with overall survival and disease-free survival in 178 HCC patients subjected to transcatheter arterial chemoembolisation (TACE). The median survival in high versus low NLR group was 8 versus 17.5 months. The 1-, 3- and 5-year overall survival rates in these groups were 42.1, 19.6 and 9.5 versus $57.3,44.1$ and $27.2 \%$, respectively ( $p<0.001$ ). Differences in disease-free survival were significant as well $(\mathrm{p}<0.001)$. Multivariate analysis confirmed NLR as a significant $(\mathrm{p}=0.04)$, independent prognostic factor for survival after TACE [56].

In patients with advanced HCC treated by hepatic arterial infusion chemotherapy, high NLR was a significant predictor of lower response rate, worse progression-free and overall survival [57]. Baseline NLR was a significant predictor of treatment response and progression-free survival after hepatic arterial infusion chemotherapy for advanced HCC [58]. In patients receiving hepatic arterial infusion chemotherapy by cisplatin and fluorouracil, response rate and overall survival were associated with NLR [59].

Dynamic changes of NLR had independent prognostic significance $(p=0.035)$ in HCC with portal vein tumour thrombosis treated by microwave ablation after transarterial chemoembolisation [60]. In 506 patients treated by thermal ablation of recurrent HCC, high pretreatment NLR $(\geq 2.14)$ was a prognostic factor for recurrence-free survival, confirmed by Cox multiple regression analysis. The 1- and 3-year recurrence rates in high versus low NLR groups were 57.9 and 82.5 versus 20.7 and $31.6 \%$. The difference was statistically significant, confirmed by $\mathrm{p}<0.001$ [61].

Pre-treatment NLR was associated with worse overall survival in early HCC after radiofrequency ablation. Post-treatment NLR was associated both with worse overall survival and recurrence in early HCC after radiofrequency ablation [62]. Similarly, NLR dynamics, but not pre-treatment NLR, was an independent prognostic factor for overall survival and recurrencefree survival in patients with small HCC treated by radiofrequency ablation [63]. In patients treated by radiofrequency ablation for HCC, post-treatment NLR was associated with recurrence and survival. Pre-treatment NLR was associated with recurrence only in patients who had HBV infection and HCC but not in those who developed HCC in association with HCV infection [64]. In unresectable HCC treated by radioembolisation, elevated NLR was an independent predictor of worse survival [65].

\subsection{Neutrophil-to-lymphocyte ratio and tumour characteristics}

NLR has been mostly assessed in correlation with survival or treatment response. However, some observations are reported on the association between systemic inflammatory response and tumour morphology in gross and microscopic level. Thus, high NLR is observed in patients having larger tumours, multiple HCC foci, higher grade of HCC and vascular invasion [29]. In few studies, the infiltration of neutrophils and macrophages in liver tumours has been assessed. Peritumoural tissues are characterised by higher ratio between neutrophils 
and T lymphocytes, and higher ratio also correlates with lower overall survival. The combination of these findings suggests that neutrophils might facilitate tumour progression. They suppress adaptive immunity by death ligand expression [66]. Correlation between NLR and PD-L1 expression in the centre of tumour but not peritumoural tissues has been described [67]. High NLR was associated with CD163-positive tumour-associated macrophages [24]. High NLR was associated with higher peritumoural but not intratumoural CD163 and IL-17expressing cells [68].

\subsection{Platelet-to-lymphocyte ratio in HCC patients}

Platelet-to-lymphocyte ratio (PLR) is another frequently assessed estimate of SIR, although fewer publications have been devoted to PLR than to NLR. Nevertheless, prognostic role of PLR has been evaluated in different aspects of HCC patient treatment, including surgery [31, $69,70]$, transplantation, sorafenib treatment, TACE and ablation techniques.

In the largest Western series enrolling 370 HCC patients, treated by surgical resection, higher preoperative PLR was identified as an independent risk factor of worse overall survival and recurrence-free survival [31]. Other research groups have confirmed the association between PLR and prognosis. Higher preoperative PLR is an independent predictor of worse overall survival in HCC patients undergoing curative liver resection $[69,70]$ as was shown in two large Eastern cohorts comprising 778 [70] and 1804 [69] patients, respectively. The significant association with overall survival $(p<0.001)$ was retained in specific subgroups, e.g., patients having cirrhosis or being positive for $\mathrm{HbsAg}$ [70]. In some studies, the associations between PLR and prognosis were statistically significant but not independent. Thus, PLR was significantly associated with disease-free and overall survival in 332 HCC patients after hepatectomy [71]. The association between higher PLR and shorter recurrence-free survival in HCC patients undergoing curative liver resection was statistically significant but not independent [70]. Finally, no association has been found in some studies. Hence, in 113 HCC patients undergoing curative resection, PLR was not confirmed as a significant prognostic factor for recurrence-free survival. NLR was found to be superior in this study [28].

PLR has been analysed in the context of liver transplantation. Already in early studies, statistically significant association was found between pretransplantation PLR and cancer recurrence after liver transplantation. In 146 patients, the recurrence-free survival was 80.7 versus 91.6\%; the difference was statistically significant as confirmed by $\mathrm{p}=0.02$ [72].

In 122 Chinese patients undergoing transarterial chemoembolisation (TACE) for HBV-related HCC, high pre-treatment PLR ( $\geq 96.13)$ was an independent, statistically significant ( $p=0.001)$ factor that predicted worse survival [73].

In 414 patients affected by recurrent HCC and treated by thermal ablation, high pre-treatment PLR ( $\geq 87.87$ ) was associated with higher risk of recurrence and worse recurrence-free survival. The 1- and 3-year recurrence rates in high versus low PLR groups were 56.0 and 79.5 versus 39.9 and $54.8 \% ; \mathrm{p}<0.05$ [74].

Regarding sorafenib-treated cases, PLR has not shown prognostic value in patients receiving sorafenib for advanced HCC. Although the negative result could be attributed to the small group size (16 patients), significant association with NLR was still confirmed [75]. 
Several meta-analyses have recently been carried out to evaluate PLR in hepatocellular carcinoma. Significant association between higher PLR and increased risk of death was reported by $\mathrm{Hu}$ and $\mathrm{Yu}$, reporting odds ratio for death 1.59; 95\% CI: 1.15-2.20 on the basis of metaanalysis of six studies with 1446 HCC patients [76]. The association between high PLR and worse overall survival was confirmed by Ma et al., Song et al., and Zhao et al. [77-79]. In a meta-analysis of nine studies including 2017 patients, high PLR was associated with poor overall survival (HR 1.63; 95\% CI: 1.42-1.88; p < 0.001) as reported by Ma et al. [77]. Evaluating 2507 patients in 11 studies, high PLR was significantly associated with worse overall survival, as reflected by hazard ratio $\mathrm{HR}=1.78 ; 95 \% \mathrm{CI}: 1.36-2.34 ; \mathrm{p}<0.001$ [78]. In a meta-analysis of 10 studies including 2315 patients, high PLR was associated with the HR for worse overall survival of 1.60; 95\% CI: 1.23-2.08; $\mathrm{p}=0.0005$ [79].

Controversial findings are reported regarding PLR and recurrence-free survival. The association has been confirmed by some research groups $[77,78]$ but denied by others. In a meta-analysis of nine studies including 2017 patients, high PLR was significantly $(\mathrm{p}<0.001)$ associated with poor recurrence-free survival: $\mathrm{HR}=1.32 ; 95 \% \mathrm{CI}$ : 1.15-1.52. In a meta-analysis of 11 studies comprising 2507 patients, elevated PLR was significantly associated with worse recurrence-free survival, as reflected by hazard ratio $\mathrm{HR}=1.82 ; 95 \% \mathrm{CI}$ : 1.56-2.13; $\mathrm{p}<0.001$. In contrast, by metaanalysis of 2315 patients in 10 studies, high PLR was not significantly associated with worse recurrence-free survival as the HR was 1.21; 95\% CI: 0.87-1.67; $\mathrm{p}=0.26$ [79].

High PLR also showed correlation with high tumour size exceeding $3 \mathrm{~cm}$, TNM stage, lymph node metastases and distant metastases [78]. The findings regarding vascular invasion are controversial again, as the association is confirmed in some studies [78] but not others [77]. No association between PLR and tumour multifocality and higher grade has been confirmed [77].

\subsection{Glasgow prognostic score in hepatocellular carcinoma}

The prognosis and treatment options of HCC patients depend not only on tumour progression but also on the extent of liver dysfunction. As a consequence, several staging systems have been proposed to predict prognosis for HCC, including Glasgow prognostic score (GPS), based on the levels of C-reactive protein and albumin (Table 1). GPS in HCC patients with hepatocellular carcinoma is an independent prognostic predictor after hepatic resection, with higher score indicating worse prognosis [80]. Thus, among 144 patients who underwent surgical resection for HCC, GPS 2 was associated with worse disease-free (HR $=2.527 ; 95 \% \mathrm{CI}$ : 1.163-5.490; $\mathrm{p}=0.019)$ and overall ( $\mathrm{HR}=8.012 ; 95 \% \mathrm{CI}: 2.818-22.784 ; \mathrm{p}<0.001)$ survival, but GPS 1 with shorter overall $(\mathrm{HR}=2.277 ; 95 \% \mathrm{CI}$ : $1.029-5.039 ; \mathrm{p}=0.042)$ survival than seen in patients preoperatively presenting with GPS 0 [80]. The independent prognostic role of GPS for overall survival was confirmed by other research teams [81] and meta-analysis [82]. Modified GPS score and dynamics of GPS score have also shown prognostic value. Thus, elevated modified GPS was associated with overall survival ( $\mathrm{HR}=2.21 ; 95 \% \mathrm{CI}$ : $1.73-2.82$; $\mathrm{p}<0.05$ ) in a meta-analysis of 2047 HCC patients [83]. Dynamics of GPS (assessed in association with hepatitis B infection status) was an independent predictor of overall survival in 247 patients treated by liver resection [84]. In addition, GPS was related to blood transfusion requirement and postoperative pulmonary complications after liver resection for HCC [85]. In patients undergoing liver transplantation for HCC, elevated GPS, reaching 1 or 2, was 


\begin{tabular}{ll}
\hline Score & Definition \\
\hline Glasgow prognostic score & \\
0 & C-reactive protein $(\mathrm{CRP})<10 \mathrm{mg} / \mathrm{L}$ AND albumin $\geq 35 \mathrm{~g} / \mathrm{L}$ \\
1 & One high-risk finding: CRP $\geq 10 \mathrm{mg} / \mathrm{L}$ OR albumin $<35 \mathrm{~g} / \mathrm{L}$ \\
2 & Both high-risk findings: CRP $\geq 10 \mathrm{mg} / \mathrm{L}$ AND albumin $<35 \mathrm{~g} / \mathrm{L}$ \\
Modified Glasgow prognostic score & CRP $\leq 10 \mathrm{mg} / \mathrm{L}$ irrespective of albumin level \\
0 & $\begin{array}{l}\text { Increased CRP on the background of normal albumin level: CRP }>10 \mathrm{mg} / \mathrm{L} \text { AND } \\
1\end{array}$ \\
& albumin $\geq 35 \mathrm{~g} / \mathrm{L}$ \\
2 & Increased CRP and hypoalbuminaemia: CRP $>10 \mathrm{mg} / \mathrm{L}$ AND albumin $<35 \mathrm{~g} / \mathrm{L}$ \\
\hline
\end{tabular}

Table 1. Glasgow prognostic score and its modifications [30].

significantly associated with poor overall $(p=0.018)$ and recurrence-free $(p=0.030)$ survival. Complex scoring system has been created on the basis of Milan criteria and GPS [86].

Regarding advanced HCC, GPS retained independent prognostic value for survival ( $\mathrm{HR}=1.410$; $95 \% \mathrm{CI}=1.060-1.874 ; \mathrm{p}=0.018$ ). In addition to the statistical significance of results, the biological survival differences by GPS were also remarkable, e.g., median survival was 480 days in patients presenting with GPS 0 and 154 days in those having GPS 1 or 2 [87]. GPS was an independent prognostic factor $(\mathrm{HR}=5.483$; 95\% CI: 2.563-11.729; $\mathrm{p}<0.001)$ for overall survival in patients undergoing sorafenib treatment: the median survival in those having GPS 0 versus elevated GPS 1 or 2 was 18.1 versus 5.2 months; $\mathrm{p}<0.001$ [88].

In HCC patients undergoing transarterial chemoembolisation, GPS was an independent prognostic factor for overall survival ( $\mathrm{HR}=1.697 ; 95 \% \mathrm{CI}$ : 1.325-2.174; $\mathrm{p}<0.001)$. It was also found to be superior to other inflammation scores as NLR, PLR or modified GPS. Based on these findings, the authors proposed a new complex score that included Child-Pugh stage, number of tumour nodules and proportion of affected liver, AFP, presence or absence of portal vein thrombosis and GPS. The new score was also a significant predictor of survival as the HR was 1.724; 95\% CI: 1.347-2.285; $\mathrm{p}<0.001$ [89].

\section{Conclusions}

HCC is a frequent and aggressive malignant tumour. Longer survival can be reached in early diagnosed and properly treated cases. In the future, diagnosis of HCC might be based on combination of radiological features and miRNA profile representing liquid biopsy. MiRNA profile can have both diagnostic and prognostic value. Thus, further lowering of the needs for core biopsy might be expected, leading to patient-friendly examination and avoiding the rare but still possible complications. After the diagnosis of HCC has been confirmed, SIR assessment by NLR and PLR represents innovative means of prognostic evaluation. 


\section{Acknowledgements}

BS was financially supported by post-doctoral research project 1.1.1.2./VIAA/1/16/242.

\section{Author details}

Ilze Strumfa ${ }^{1 *}$, Dzeina Mezale ${ }^{1}$, Boriss Strumfs ${ }^{2}$, Andrejs Vanags $^{3}$, Arturs Kalva ${ }^{1}$, Dainis Balodis ${ }^{1}$, Ilze Fridrihsone ${ }^{1}$, Arnis Abolins ${ }^{1}$ and Janis Gardovskis ${ }^{3}$

*Address all correspondence to: ilze.strumfa@rsu.lv

1 Department of Pathology, Riga Stradins University, Riga, Latvia

2 Latvian Institute of Organic Synthesis, Riga, Latvia

3 Department of Surgery, Riga Stradins University, Riga, Latvia

\section{References}

[1] Siegel RL, Miller KD, Jemal A. Cancer statistics, 2016. CA: A Cancer Journal for Clinicians. 2016;66(1):7-30

[2] Shah C, Mramba LK, Bishnoi R, Bejjanki H, Chhatrala HS, Chandana SR. Survival differences among patients with hepatocellular carcinoma based on the stage of disease and therapy received: Pre and post sorafenib era. Journal of Gastrointestinal Oncology. 2017;8(5):789-798

[3] Filingeri V, Francioso S, Sforza D, Santopaolo F, Oddi FM, Tisone G. A retrospective analysis of 1.011 percutaneous liver biopsies performed in patients with liver transplantation or liver diseases: Ultrasonography can reduce complications? European Review for Medical and Pharmacological Sciences. 2016;20(17):3609-3617

[4] Tan Y, Ge G, Pan T, Wen D, Chen L, Yu X, Zhou X, Gan J. A serum microRNA panel as potential biomarkers for hepatocellular carcinoma related with hepatitis B virus. PLoS One. 2014;9(9):e107986

[5] Loosen SH, Schueller F, Trautwein C, Roy S, Roderburg C. Role of circulating microRNAs in liver diseases. World Journal of Hepatology. 2017;9(12):586-594

[6] Qi P, Cheng SQ, Wang H, Li N, Chen YF, Gao CF. Serum microRNAs as biomarkers for hepatocellular carcinoma in Chinese patients with chronic hepatitis B virus infection. PLoS One. 2011;6(12):e28486

[7] Sohn W, Kim J, Kang SH, Yang SR, Cho JY, Cho HC, Shim SG, Paik YH. Serum exosomal microRNAs as novel biomarkers for hepatocellular carcinoma. Experimental \& Molecular Medicine. 2015;47:e184 
[8] Ali HEA, Abdel Hameed R, Effat H, Ahmed EK, Atef AA, Sharawi SK, Ali M, Abd Elmageed ZY, Abdel Wahab AH. Circulating microRNAs panel as a diagnostic tool for discrimination of HCV-associated hepatovcellular carcinoma. Clinics and Research in Hepatology and Gastroenterology. 2017;41(4):e51-e62

[9] Zhang Y, Li T, Qiu Y, Zhang T, Guo P, Ma X, Wei Q, Han L. Serum microRNA panel for early diagnosis of the onset of hepatocellular carcinoma. Medicine (Baltimore). 2017;96(2):e5642

[10] Zekri AN, Youssef AS, El-Desouky ED, Ahmed OS, Lotfy MM, Nassar AA, Bahnassey AA. Serum microRNA panels as potential biomarkers for early detection of hepatocellular carcinoma on top of HVCV infection. Tumour Biology. 2016;37(9):12273-12286

[11] Khairy A, Hamza I, Shaker O, Yosry A. Serum miRNA panel in Egyptian patients with chronic hepatitis C related hepatocellular carcinoma. Asian Pacific Journal of Cancer Prevention. 2016;17(5):2699-2703

[12] Bimonte S, Leongito M, Barbieri A, Del Vecchio V, Falco M, Giudice A, Palaia R, Albino V, Di Giacomo R, Petrillo A, Granata V, Izzo F. The therapeutic targets of miRNA in hepatic cancer stem cells. Stem Cells International. 2016;2016:1065230

[13] Ohki S, Shibata M, Gonda K, Machida T, Shimura T, Nakamura I, Ohtake T, Koyama Y, Suzuki S, Ohto H, Takenoshita S. Circulating myeloid-deribved suppressor cells are increased and correlate to immune suppression, inflammation and hypoproteinemia in patients with cancer. Oncology Reports. 2012;28(2):453-458

[14] Rutkowski MR, Svoronos N, Perales-Puchalt A, Conejo-Garcia JR. The tumor macroenvironment: Cancer promoting networks beyond tumor beds. Advances in Cancer Research. 2015;128:235-262

[15] Olsson AK, Cederval J. NETosis in cancer - Platelet-neutrophil crosstalk promotes tumor-associated pathology. Frontiers in Immunology. 2016;7:373

[16] Cederval J, Dimberg A, Olsson AK. Tumor-induced local and systemic impact on blood vessel function. Mediators of Inflammation. 2015;2015:418290

[17] Mohri Y, Tanaka K, Toiyama Y, Ohi M, Yasuda H, Inoue Y, Kusunoki M. Impact of preoperative neutrophil to lymphocyte ratio and postoperative infectious complications on survival after curative gastrectomy for gastric cancer: A single institutional cohort study. Medicine (Baltimore). 2016;95(11):e3125. DOI: 10.1097/MD0000000000003125

[18] De Visser KE, Coussens LM. The interplay between innate and adaptive immunity regulates cancer development. Cancer Immunology, Immunotherapy. 2005;54(11):1143-1152

[19] Abdel-RazikA, Mousa N, BesheerTA, Eissa M, Elhelaly R, Arafa M, El-Wakeel N, Eldars W. Neutrophil to lymphocyte ratio as a reliable marker to predict insulin resistance and fibrosis stage in chronic hepatitis C virus infection. Acta Gastroenterologica Belgica. 2015;78(4):386-392

[20] Biyik M, Ucar R, Solak Y, Gungor G, Polat I, Gaipov A, Cakir OO, Ataseven H, Demir A, Turk S, Polat H. Blood neutrophil-to-lymphocyte ratio independently predicts survival 
in patients with liver cirrhosis. European Journal of Gastroenterology \& Hepatology. 2013;25(4):435-441

[21] Qi X, Li J, Deng H, Li H, Su C, Guo X. Neutrophil-to-lymphocyte ratio for the prognostic assessment of hepatocellular carcinoma: A systematic review and meta-analysis of observational studies. Oncotarget. 2016;7(29):45283-45301

[22] Sun XD, Shi XJ, Chen YG, Wang CL, Ma Q, Lv GY. Elevated preoperative neutrophillymphocyte ratio is associated with poor prognosis in hepatocellular carcinoma patients treated with liver transplantation: A meta-analysis. Gastroenterology Research and Practice. 2016;2016:4743808

[23] Xiao WK, Chen D, Li SQ, Fu SJ, Peng BG, Liang LJ. Prognostic significance of neutrophillymphocyte ratio in hepatocellular carcinoma: A meta-analysis. BMC Cancer. 2014;14:117

[24] Mano Y, Shirabe K, Yamashita Y, Harimoto N, Tsujita E, Takeishi K, Aishima S, Ikegami T, Yoshizumi T, Yamanaka T, Maehara Y. Preoperative neutrophil-to-lymphocyte ratio is a predictor of survival after hepatectomy for hepatocellular carcinoma: A retrospective analysis. Annals of Surgery. 2013;258(2):301-305

[25] Okamura Y, Ashida R, Ito T, Sugiura T, Mori K, Uesaka K. Preoperative neutrophil to lymphocyte ratio and prognostic nutritional index predict overall survival after hepatectomy for hepatocellular carcinoma. World Journal of Surgery. 2015;39(6):1501-1509

[26] Huang GQ, Zhu GQ, Liu YL, Wang LR, Braddock M, Zheng MH, Zhou MT. Stratified neutrophil-to-lymphocyte ratio accurately predict mortality risk in hepatocellular carcinoma patients following curative liver transplantation. Oncotarget. 2016;7(5):5429-5439

[27] Ji F, Fu S, Guo Z, Pang H, Chen D, Wang X, Ju W, Wang D, He X, Hua Y, Peng B. Prognostic significance of preoperative aspartate aminotransferase to neutrophil ratio index in patients with hepatocellular carcinoma after hepatic resection. Oncotarget. 2016;7(44):72276-72289

[28] Yamamura K, Sugimoto H, Kanda M, Yamada S, Nomoto S, Nakayama G, Fujii T, Koike M, Fujiwara M, Kodera Y. Comparison of inflammation-based prognostic scores as predictors of tumor recurrence in patients with hepatocellular carcinoma after curative resection. Journal of Hepato-Biliary-Pancreatic Sciences. 2014;21(9):682-688

[29] Hung HC, Lee JC, Cheng CH, Wu TH, Wang YC, Lee CF, Wu TJ, Chou HS, Chan KM, Lee WC. Impact of neutrophil to lymphocyte ratio on survival for hepatocellular carcinoma after curative resection. Journal of Hepato-Biliary-Pancreatic Sciences. 2017. DOI: 10.1002/jhbp.498 [Epub ahead of print]

[30] Strumfa I, Bogdanova T, Kalva A, Strumfs B, Rumba R, Vanags A, Drike I, Mezale D, Abolins A, Jakovlevs A, Balodis D, Gardovskis J. Systemic inflammatory reaction in gastric cancer: Biology and practical implications of neutrophil to lymphocyte ratio, Glasgow prognostic score and related parameters. In: Mozsik G, editor. Gastric Cancer. InTech; 2017. pp. 143-197. DOI: 10.5772/intechopen.69723

[31] Zheng J, Seier K, Gonen M, Balachandran VP, Kingham TP, D'Angelica MI, Allen PJ, Jarnagin WR, DeMatteo RP. Utility of serum inflammatory markers for predicting 
microvascular invasion and survival for patients with hepatocellular carcinoma. Annals of Surgical Oncology. 2017. DOI: 10.1245/s10434-017-6060-7 [Epub ahead of print]

[32] Okamura Y, Sugiura T, Ito T, Yamamoto Y, Ashida R, Mori K, Uesaka K. Neutrophil to lymphocyte ratio as an indicator of the malignant behaviour of hepatocellular carcinoma. The British Journal of Surgery. 2016;103(7):891-898

[33] Liao R, Tang ZW, Li DW, Luo SQ, Huang P, Du CY. Preoperative neutrophil-to-lymphocyte ratio predicts recurrence of patients with single-nodule small hepatocellular carcinoma following curative resection: A retrospective report. World Journal of Surgical Oncology. 2015;13:265

[34] Lu SD, Wang YY, Peng NF, Peng YC, Zhong JH, Qin HG, Xiang BD, You XM, Ma L, Li LQ. Preoperative ratio of neutrophils to lymphocytes predicts postresection survival in selected patients with early or intermediate stage hepatocellular carcinoma. Medicine (Baltimore). 2016;95(5):e2722

[35] Goh BK, Kam JH, Lee SY, Chan CY, Allen JC, Jeyaraj P, Cheow PC, Chow PK, Ooi LL, Chung AY. Significance of neutrophil-to-lymphocyte ratio, platelet-to-lymphocyte ratio and prognostic nutrition index as preoperative predictors of early mortality after liver resection for huge $(\geq 10 \mathrm{~cm})$ hepatocellular carcinoma. Journal of Surgical Oncology. 2016;113(6):621-627

[36] Shimoda M, Tago K, Shiraki T, Mori S, Kato M, Aoki T, Kubota K. Risk factors for early recurrence of single lesion hepatocellular carcinoma after curative resection. World Journal of Surgery. 2016;40(10):2466-2471

[37] Chan AW, Chan SL, Wong GL, Wong vW, Chong CC, Lai PB, Chan HL, To KF. Prognostic nutritional index (PNI) predicts tumor recurrence of very early/early stage hepatocellular carcinoma after surgical resection. Annals of Surgical Oncology. 2015;22(13):4138-4148

[38] Li SH, Wang QX, Yang ZY, Jiang W, Li C, Sun P, Wei W, Shi M, Guo RP. Prognostic value of the neutrophil-to-lymphocyte ratio for hepatocellular carcinoma patients with portal/ hepatic vein tumor thrombosis. World Journal of Gastroenterology. 2017;23(17):3122-3132

[39] Peng W, Li C, Wen TF, Yan LN, Li B, Wang WT, Yang JY, Xu MQ. Neutrophil to lymphocyte ratio changes predict small hepatocellular carcinoma survival. The Journal of Surgical Research. 2014;192(2):402-408

[40] Bertuzzo VR, Cescon M, Ravaioli M, Grazi GL, Ercolani G, Del Gaudio M, Cuccheti A, D'Errico-Grigioni A, Golfieri R, Pinna AD. Analysis of factors affecting recurrence of hepatocellular carcinoma after liver transplantation with a special focus on inflammation markers. Transplantation. 2011;91(11):1279-1285

[41] Fu SJ, Ji F, Han M, Chen MG, Wang XP, Ju WQ Zhao Q Wu LW, Ren QQ Guo ZY, Wang DP, Zhu XF, Ma Y, He XS. Prognostic value of combined preoperative fibrinogen and neutrophil-lymphocyte ratio in patients with hepatocellular carcinoma after liver transplantation. Oncotarget. 2017;8(3):4301-4312 
[42] Limaye AR, Clark V, Soldevila-Pico C, Morelli G, Suman A, Firpi R, Nelson DR, Cabrera R. Neutrophil-lymphocyte ratio predicts overall and recurrence-free survival after liver transplantation for hepatocellular carcinoma. Hepatology Research. 2013;43(7):757-764

[43] Wang W, Ye Y, Wang T, Zhang F, Geng L, Yu J, Zhou L, Yan S, Zheng S. Prognostic prediction of male recipients selected for liver transplantation: With special attention to neutrophil to lymphocyte ratio. Hepatology Research. 2016;46(9):899-907

[44] Harimoto N, Yoshizumi T, Shimagaki T, Nagatsu A, Motomura T, Harada N, Okabe H, Itoh S, Ikegami T, Uchiyama H, Soejima Y, Maehara Y. Inflammation-based prognostic score in patients with living donor liver transplantation for hepatocellular carcinoma. Anticancer Research. 2016;36(10):5537-5542

[45] Xiao GQ, Yang JY, Yan LN. Combined Hangzhou criteria with neutrophil-lymphocyte ratio is superior to other criteria in selecting liver transplantation candidates with HBVrelated hepatocellular carcinoma. Hepatobiliary \& Pancreatic Diseases International. 2015;14(6):588-595

[46] Halazun KJ, Najjar M, Abdelmessih RM, Samstein B, Griesemer AD, Guarrera JV, Kato T, Verna EC, Emond JC, Brown Jr RS. Recurrence after liver transplantation for hepatocellular carcinoma: A new MORAL to the story. Annals of Surgery. 2017;265(3):557-564

[47] Na GH, Kim DG, Han JH, Kim EY, Lee SH, Hong TH, You YK. Inflammatory markers as selection criteria of hepatocellular carcinoma in living-donor liver transplantation. World Journal of Gastroenterology. 2014;20(21):6594-6601

[48] Parisi I, Tsochatzis E, Wijewantha H, Rodriguez-Peralvarez M, De Luca L, Manousou P, Fatourou E, Pieri G, Papastergiou V, davies N, Yu D, Luong T, Dhillon AP, Thorburn D, Patch D, O'Beirne J, Meyer T, Burroughs AK. Inflammation-based scores do not predict post-transplant recurrence of hepatocellular carcinoma in patients within Milan criteria. Liver Transplantation. 2014;20(11):1327-1335

[49] Shindoh J, Sugawara Y, Nagata R, Kaneko J, Tamura S, Aoki T, Sakamoto Y, Hasegawa K, Tanaka T, Kokudo N. Evaluation methods for pretransplant oncologic markers and their prognostic impacts in patient undergoing living donor liver transplantation for hepatocellular carcinoma. Transplant International. 2014;27(4):391-398

[50] Sullivan KM, Groeschl RT, Turaga KK, Tsai S, Christians KK, White SB, Rilling WS, Pilgrim CH, Gamblin TC. Neutrophil-to-lymphocyte ratio as a predictor of outcomes for patients with hepatocellular carcinoma: A western perspective. Journal of Surgical Oncology. 2014;109(2):95-97

[51] Bruix J, Cheng AL, Meinhardt G, Nakajima K, De Sanctis Y, Llovet J. Prognostic factors and predictors of sorafenib benefit in patients with hepatocellular carcinoma: Analysis of two phase II studies. Journal of Hepatology. 2017. DOI: 10.1016/j.jhep.2017.06.026 [Epub ahead of print]

[52] Howell J, Pinato DJ, Ramaswami R, Arizumi T, Ferrari C, Gibbin A, Burlone ME, Guaschino G, Toniutto P, Black J, Sellers L, Kudo M, Pirisi M, Sharma R. Integration of 
the cancer-related inflammatory response as a stratifying biomarker of survival in hepatocellular carcinoma treated with sorafenib. Oncotarget. 2017;8(22):36161-36170

[53] Wei K, Wang M, Zhang W, Mu H, Song TQ. Neutrophil-lymphocyte ratio as a predictor of outcomes for patients with hepatocellular carcinoma undergoing TAE combined with Sorafenib. Medical Oncology. 2014;31(6):969

[54] Zheng YB, Zhao W, Liu B, Lu LG, He X, Huang JW, Li Y, Hu BS. The blood neutrophil-tolymphocyte ratio predicts survival in patients with advanced hepatocellular carcinoma receiving sorafenib. Asian Pacific Journal of Cancer Prevention. 2013;14(9):5527-5531

[55] Taussig MD, Irene Koran ME, Mouli SK, Ahmad A, Geevarghese S, Baker JC, Lipnik AJ, Banovac F, Brown DB. Neutrophil to lymphocyte ratio predicts disease progression following intra-arterial therapy of hepatocellular carcinoma. HPB: The Official Journal of the International Hepato Pancreato Biliary Association. 2017;19(5):458-464

[56] Xu X, Chen W, Zhang L, Miao R, Zhou Y, Wan Y, Dong Y, Liu C. Prognostic significance of neutrophil to lymphocyte ratio in patients with hepatocellular carcinoma after transcatheter arterial chemoembolization. Chinese Medical Journal. 2014;127(24):4204-4209

[57] Terashima T, Yamashita T, Iida N, Yamashita T, Nakagawa H,Arai K, Kitamura K, KagayaT, Sakai Y, Mizukoshi E, Honda M, Kaneko S. Blood neutrophil to lymphocyte ratio as a predictor in patients with advanced hepatocellular carcinoma treated with hepatic arterial infusion chemotherapy. Hepatology Research. 2014. DOI: 10.1111/hepr.12436

[58] TsunematsuS,Suda G, YamasakiK,KimuraM,Takaaki I,UmemuraM,ItoJ,SatoF,NakaiM, Sho T, Morikawa K, Ogawa K, Kamiyama T, Taketomi A, Sakamoto N. Combination of neutrophil-to-lymphocyte ratio and early des-gamma-carboxyprothrombin change ratio as a useful predictor of treatment response for hepatic arterial infusion chemotherapy against advanced hepatocellular carcinoma. Hepatology Research. 2017;47(6):533-541

[59] Tajiri K, Kawai K, Minemura M, Yasumura S, Hosokawa A, Kawabe H, Tomizawa G, Sugiyama T. Neutrophil/lymphocyte ratio as a prognostic indicator of hepatic arterial infusion chemotherapy with arterial cisplatin plus continuous 5-fluorouracil. Hepatology Research. 2015;45(7):755-763

[60] Long J, Zheng JS, Sun B, Lu N. Microwave ablation of hepatocellular carcinoma with portal vein tumor thrombosis after transarterial chemoembolization: A prospective study. Hepatology International. 2016;10(1):175-184

[61] Li X, Han Z, Cheng Z, Yu J, Liu S, Yu X, Liang P. Preoperative neutrophil-to-lymphocyte ratio is a predictor of recurrence following thermal ablation for recurrent hepatocellular carcinoma: A retrospective analysis. PLoS One. 2014;9(10):e110546

[62] Chen TM, Lin CC, Huang PT, Wen CF. Neutrophil-to-lymphocyte ratio associated with mortality in early hepatocellular carcinoma patients after radiofrequency ablation. Journal of Gastroenterology and Hepatology. 2012;27(3):553-561

[63] Dan J, Zhang Y, Peng Z, Huang J, Gao H, Xu L, Chen M. Postoperative neutrophil-tolymphocyte ratio change predicts survival of patients with small hepatocellular carcinoma undergoing radiofrequency ablation. PLoS One. 2013;8(3):e58184 
[64] Tajiri K, Baba H, Kawai K, Minemura M, Yasumura S, Takahara T, Sugiyama T. Neutrophilto-lymphocyte ratio predicts recurrence after radiofrequency ablation in hepatitis B virus infection. Journal of Gastroenterology and Hepatology. 2016;31(7):1291-1299

[65] Sukato DC, Tohme S, Chalhoub D, Han K, Zajko A, Amesur N, Orons P, Marsh JW, Geller DA, Tsung A. The prognostic role of neutrophil-to-lymphocyte ratio in patients with unresectable hepatocellular carcinoma treated with radioembolization. Journal of Vascular and Interventional Radiology. 2015;26(6):816-824

[66] He G, Zhang H, Zhou J, Wang B, Chen Y, Kong Y, Xie X, Wang X, Fei R, Wei L, Chen H, Zeng $H$. Peritumoural neutrophils negatively regulate adaptive immunity via the PD-L1/PD-1 signalling pathway in hepatocellular carcinoma. Journal of Experimental \& Clinical Cancer Research. 2015;34:141

[67] Wang Q, Blank S, Fiel MI, Kadri H, Luan W, Warren L, Zhu A, Deaderick PA, Sarpel U, Labow DM, Hiotis SP. The severity of liver fibrosis influences the prognostic value of inflammation-based scores in hepatitis B-associated hepatocellular carcinoma. Annals of Surgical Oncology. 2015;22(Suppl 3):S1125-S1132

[68] Motomura T, ShirabeK, Mano Y, Muto J, Toshima T, Umemoto Y, Fukuhara T, UchiyamaH, Ikegami T, Yoshizumi T, Soejima Y, Maehara Y. Neutrophil-lymphocyte ratio reflects hepatocellular carcinoma recurrence after liver transplantation via inflammatory microenvironment. Journal of Hepatology. 2013;58(1):58-64

[69] Huang GQ, Zheng JN, Zou TT, Chen YR, Shi KQ, PouckeSV, Cheng Z, Ruan LY, Zheng MH. Stratified platelet-to lymphocyte ratio: A novel target for prognostic prediction of hepatocellular carcinoma after curative liver resection. Journal of Clinical and Translational Hepatology. 2017;5(1):35-42

[70] Yang HJ, Jiang JH, Liu QA, Zhou CM, Du YF, Wu T, Chen NZ, Xiang BD. Preoperative platelet-to-lymphocyte ratio is a valuable prognostic biomarker in patients with hepatocellular carcinoma undergoing curative liver resection. Tumour Biology. 2017;39(6). DOI: 1010428317707375

[71] Shen SL, Fu SJ, Chen B, Kuang M, Li SQ, Hua YP, Liang LJ, Guo P, Hao Y, Peng BG. Preoperative aspartate aminotransferase to platelet ratio is an independent prognostic factor for hepatitis B-induced hepatocellular carcinoma after hepatic resection. Annals of Surgical Oncology. 2014;21(12):3802-3809

[72] Lai Q, Castro Santa E, Rico Juri JM, Pinheiro RS, Lerut J. Neutrophil and platelet-tolymphocyte ratio as new predictors of dropout and recurrence after liver transplantation for hepatocellular cancer. Transplant International. 2014;27(1):32-41

[73] Tian XC, Liu XL, Zeng FR, Chen Z, Wu DH. Platelet-to-lymphocyte ratio acts as an independent risk factor for patients with hepatitis B virus-related hepatocellular carcinoma who received transarterial chemoembolization. European Review for Medical and Pharmacological Sciences. 2016;20(11):2302-2309

[74] Li X, Han Z, Cheng Z, Yu J, Yu X, Liang P. Clinical significance of the preoperative platelet-to-lymphocyte ratio in recurrent hepatocellular carcinoma after thermal ablation: A retrospective analysis. International Journal of Hyperthermia. 2015;31(7):758-763 
[75] Shiozawa K, Watanabe M, Ikehara T, Matsukiyo Y, Kogame M, Shinohara M, Kikuchi Y, Igarashi Y, Sumino Y. Plasma biomarkers as predictive factors for advanced hepatocellular carcinoma with sorafenib. [article in Japanese]. Gan to Kagaku Ryoho. 2016;43(7): 63-867

[76] Hu DH, Yu SM. Association between platelet to lymphocyte ratio (PLR) and overall survival (OS) of hepatocellular carcinoma (HCC): A meta-analysis. Cellular and Molecular Biology (Noisy-le-Grand, France). 2017;63(8):30-32

[77] Ma W, Zhang P, Qi J, Gu L, Zang M, Yao H, Shi X, Wang C, Jiang Y. Prognostic value of platelet to lymphocyte ratio in hepatocellular carcinoma: A meta-analysis. Scientific Reports. 2016;6:35378

[78] Song W, Wang K, Zhong FP, Fan YW, Peng L, Zou SB. Clinicopathological and prognostic significance of platelet-to-lymphocyte ratio in patients with hepatocellular carcinoma. Oncotarget. 2016;7(49):81830-81838

[79] Zhao Y, Si G, Zhu F, Hui J, Cai S, Huang C, Cheng S, Fathy AH, Xiang Y, Li J. Prognostic role of platelet to lymphocyte ratio in hepatocellular carcinoma: A systematic review and meta-analysis. Oncotarget. 2017;8(14):22854-22862

[80] Shiba H, Horiuchi T, Sakamoto T, Furukawa K, Shirai Y, Iida T, Fujiwara Y, Haruki K, Yanaga K. Glasgow prognostic score predicts therapeutic outcome after hepatic resection for hepatocellular carcinoma. Oncology Letters. 2017;14(1):293-298

[81] Abe T, Tashiro H, Kobayashi T, Hattori M, Kuroda S, Ohdan H. Glasgow prognostic score and prognosis after hepatectomy for hepatocellular carcinoma. World Journal of Surgery. 2017;41(7):1860-1870

[82] Li MX, Bi XY, Li ZY, Huang Z, Han Y, Zhou JG, Zhao JJ, Zhang YF, Zhao H, Cai JQ. Prognostic role of Glasgow prognostic score in patients with hepatocellular carcinoma: A systematic review and meta-analysis. Medicine (Baltimore). 2015;94(49):e2133

[83] Chen H, Hu N, Chang P, Kang T, Han S, Lu Y, Li M. Modified Glasgow prognostic score might be a prognostic factor for hepatocellular carcinoma: A meta-analysis. Panminerva Medica. 2017;59(4):302-307

[84] Pang S, Zhou Z, Yu X, Wei S, Chen Q, Nie S, Liang X, Liu L. The predictive value of integrated inflammation scores in the survival of patients with resected hepatocellular carcinoma: A retrospective cohort study. International Journal of Surgery. 2017;42:170-177

[85] Fujiwara Y, Shiba H, Furukawa K, Iida T, Haruki K, Gocho T, Wakiyama S, Hirohara S, Ishida Y, Misawa T, Ohashi T, Yanaga K. Glasgow prognostic score is related to blood transfusion requirements and post-operative complications in hepatic resection for hepatocellular carcinoma. Anticancer Research. 2010;30(12):5129-5136

[86] Abe T, Tashiro H, Hattori M, Kuroda S, Tahara H, Ohira M, Kobayashi T, Ide K, Ishiyama K, Ohdan H. Prediction of long-term survival by using the Glasgow prognostic score in patients with hepatocellular carcinoma after liver transplantation. Hepatology Research. 2016;46(7):622-633 
[87] Aino H, Sumie S, Niizeki T, Kuromatsu R, Tajiri N, Nakano M, Satani M, Okamura S, Shimose S, Miyahara K, Torimura T. The systemic inflammatory response as a prognostic factor for advanced hepatocellular carcinoma with extrahepatic metastasis. Molecular and Clinical Oncology. 2016;5(1):83-88

[88] Morimoto M, Numata K, Moriya S, Kondo M, Nozaki A, Morioka Y, Maeda S, Tanaka K. Inflammation-based prognostic score for hepatocellular carcinoma patients on sorafenib treatment. Anticancer Research. 2012;32(2):619-623

[89] Zhou DS, Xu L, Luo YL, He FY, Huang JT, Zhang YJ, Chen MS. Inflammation scores predict survival for hepatitis B virus-related hepatocellular carcinoma patients after transarterial chemoembolization. World Journal of Gastroenterology. 2015;21(18):5582-5590 
